\title{
Corrigendum: Open carpal release using local anesthesia without a tourniquet: Does bleeding tendency affect the outcome?
}

\author{
Seongwon Lee ${ }^{1}$, Sangho $\mathrm{Oh}^{2}$, Daegu Son ${ }^{1}$ \\ ${ }^{1}$ Department of Plastic and Reconstructive Surgery, Keimyung University School of Medicine, Daegu; ${ }^{2}$ W-Hospital, Daegu, Korea
}

Arch Plast Surg 2020;47:597-603

https://doi.org/10.5999/aps.2020.01732

We have noticed two errors in our published paper above. The corrected version is as follows.

Correction of Title

Open carpal tunnel release using local anesthesia without tourniquet: Does bleeding tendency affect the outcome?

Correction of Running Title

Open carpal tunnel release without tourniquet

We sincerely apologize for the inconvenience that this may have caused. 
